Published in final edited form as:

Brain Stimul. 2013 September ; 6(5): 737-739. doi:10.1016/j.brs.2013.03.008.

\title{
Tractography-activation models applied to subcallosal cingulate deep brain stimulation
}

\author{
J. Luis Lujan ${ }^{1}$, Ashutosh Chaturvedi ${ }^{1}$, Ki Sueng Choi ${ }^{2}$, Paul E. Holtzheimer ${ }^{3,4}$, Robert E. \\ Gross $^{2,5,6}$, Helen S. Mayberg ${ }^{3,6}$, and Cameron C. McIntyre ${ }^{1}$ \\ ${ }^{1}$ Department of Biomedical Engineering, Cleveland Clinic Foundation, Cleveland, $\mathrm{OH}$ \\ ${ }^{2}$ Coulter Department of Biomedical Engineering, Georgia Institute of Technology; Atlanta, GA \\ ${ }^{3}$ Department of Psychiatry and Behavioral Sciences, Emory University School of Medicine, \\ Atlanta, GA \\ ${ }^{4}$ Departments of Psychiatry and Surgery, Geisel School of Medicine at Dartmouth, Lebanon, NH \\ ${ }^{5}$ Department of Neurosurgery, Emory University School of Medicine, Atlanta, GA \\ ${ }^{6}$ Department of Neurology, Emory University School of Medicine, Atlanta, GA
}

\begin{abstract}
Deep brain stimulation (DBS) of the subcallosal cingulate white matter (SCCWM) is an experimental therapy for major depressive disorder (MDD). The specific axonal pathways that mediate the anti-depressant effects of DBS remain unknown. Patient-specific tractographyactivation models (TAMs) are a new tool to help identify pathways modulated by DBS. TAMs consist of four basic components: 1) anatomical and diffusion-weighted imaging data acquired on the patient; 2) probabilistic tractography from the brain region surrounding the implanted DBS electrode; 3) finite element models of the electric field generated by the patient-specific DBS parameter settings; 4) application of the DBS electric field to multi-compartment cable models of axons, with trajectories defined by the tractography, to predict action potential generation in specific pathways. This study presents TAM predictions from DBS of the SCCWM in one MDD patient. Our findings suggest that small differences in electrode location can generate substantial differences in the directly activated pathways.
\end{abstract}

\section{Keywords}

depression; axon; white matter; cortex; cingulum; accumbens

(c) 2XXX Elsevier Inc. All rights reserved.

Correspondence: Cameron C. McIntyre, Ph.D. Department of Biomedical Engineering, Case Western Reserve University, 10900 Euclid Avenue, Cleveland, OH 44106, ccm4@ case.edu.

Disclosures: Intellectual Property: Boston Scientific Neuromodulation (JLL, AC, CCM), St Jude Medical Neuromodulation (HSM). Equity Interest: Autonomic Technologies (CCM), Neuros Medical (CCM), Surgical Information Sciences (CCM). Paid Consultants: Boston Scientific Neuromodulation (CCM, REG), St. Jude Medical Neuromodulation (PEH, REG, HSM), Medtronic/Lilly (REG), Cervel Neurotech (PEH); Johnson \& Johnson (PEH).

Publisher's Disclaimer: This is a PDF file of an unedited manuscript that has been accepted for publication. As a service to our customers we are providing this early version of the manuscript. The manuscript will undergo copyediting, typesetting, and review of the resulting proof before it is published in its final citable form. Please note that during the production process errors may be discovered which could affect the content, and all legal disclaimers that apply to the journal pertain. 


\section{Introduction}

Deep brain stimulation (DBS) represents a promising experimental therapy for patients with depression [1, 2]. However, the target axonal pathways and most appropriate stimulation parameter settings responsible for the purported therapeutic response to DBS are not completely understood. Clinical DBS electrodes are implanted in white matter regions that connect multiple gray matter structures implicated in the origin of neuropsychiatric disorders. Diffusion-weighted imaging (DWI) in humans $[3,4]$ and anatomical tracing in non-human primates [5] have documented a wide range of different pathways that may be stimulated by DBS for the treatment of depression. However, we propose that the next generation analyses should include both anatomical tractography and electrical activation representations of the therapy [6]. Therefore, we created a patient-specific tractographyactivation model (TAM) to investigate DBS of the subcallosal cingulate white matter (SCCWM) in a patient with major depressive disorder (MDD).

\section{Methods}

Ethical approval from the Emory University institutional review board and informed written consent from the patient were obtained prior to enrollment in a clinical trial evaluating SCCWM DBS [2]. The patient was implanted with quadripolar DBS electrodes which were $1.4 \mathrm{~mm}$ in diameter and consisted of one $3 \mathrm{~mm}$ long contact tip followed by three $1.5 \mathrm{~mm}$ long contacts, separated by $1.5 \mathrm{~mm}$ non-conductive spacing (St. Jude Medical, St. Paul, MN) (Fig. 1A). The results of this study compare stimulation through either contact \#1 or contact \#3. These contacts were independently evaluated with intra-operative testing while the patient was blind to the active contact. In prior reports [1,2], approximately two-thirds of patients experience positive effects from acute SCCWM DBS, including a sense of increased alertness, less psychic pain, decreased heaviness, and increased interest/ motivation. It is unclear if these effects can explicitly predict long-term antidepressant response to SCCWM DBS. The choice of contact used for long-term stimulation was primarily based on anatomical location within the SCCWM, as previously described [2]. Long-term therapeutic benefit in this patient was achieved via bilateral monopolar stimulation through contact \#3 with $8 \mathrm{~mA}, 90 \mu$ s pulses delivered at $130 \mathrm{~Hz}$ on both sides of the brain. Prior to surgery the patient had a score of 23 on the Hamilton Depression Rating Scale (HDRS17), which reduced to 5 after 1 year of stimulation through contact \#3. This classified the patient as in remission, which has been sustained.

\section{Imaging Data}

A T1- weighted MRI at 3T was acquired pre-operatively (voxel size of $1 \times 1 \times 1 \mathrm{~mm}$ ), along with diffusion weighted images (DWI) $\left(b=1000 \mathrm{sec} / \mathrm{mm}^{2}\right)$ acquired along 64 gradients (voxel sixe $2 \times 1.94 \times 1.94 \mathrm{~mm}$ ). DWI data were pre-processed with FSL to correct for motion artifacts and Eddy currents in the gradient coils [7]. A post-operative CT image was also acquired (voxel sixe $0.48 \times 0.48 \times 0.62 \mathrm{~mm}$ ) for DBS electrode localization. All imaging data was co-registered to an image with no diffusion weighting $\left(b=0 \mathrm{sec} / \mathrm{mm}^{2}\right)$ to determine the affine transformations across the different image data sets.

\section{Tractography-Activation Models}

We used TAMs to compare the stimulation effects at the most therapeutic contact (\#3) versus a non-therapeutic contact (\#1). TAMs combine tractography models with electric field models to predict direct axonal activation in response to specific DBS parameter settings in the patient $[6,8]$. A seed volume $(18 \times 24 \times 28 \mathrm{~mm})$ encompassing the SCC region and DBS electrode was defined in the patient's structural MRI. Probabilistic tractography was performed from the seed volume with a uniform distribution of 100 
streamlines from each DWI voxel using FSL [7]. These trajectories were used to define the geometry of 110,000 multi-compartment cable models of myelinated axons [9] (Fig. 1C). Each axon model was defined using a fiber diameter of $5.7 \mu \mathrm{m}$ and thereby provided a "worst case" estimate of the spread of axonal activation during SCCWM DBS.

A finite element model (FEM) of the DBS electrode and surrounding brain tissue was created to simulate the voltage distribution generated in the brain by stimulation through each individual electrode contact [10]. The diffusion tensors, calculated at each voxel in the DWI, were transformed into conductivity tensors and integrated into the FEM to account for the anisotropy and inhomogeneity of the patient brain [11]. The extracellular voltage distribution interpolated along each axon model trajectory was used to simulate the change in transmembrane potential induced by each DBS pulse (Fig. 1D) [12]. Axons which received sufficient polarization from DBS responded with action potential generation and were deemed to be directly activated by the given stimulation parameter settings (Fig. $1 \mathrm{E}, \mathrm{F}, \mathrm{G}, \mathrm{H})$.

\section{Results}

SCCWM DBS TAMs predicted robust activation of numerous pathways. For simplicity, Figure 1 shows unilateral TAM results for left side stimulation, as similar activation patterns were seen with right side simulation. DBS through the most therapeutic electrode contact (contact \#3) (8 mA) resulted in direct activation of 4,131 axon models that primarily projected to/from brain regions associated with the ventromedial pre-frontal cortex (vmPFC) (i.e. BA10), nucleus accumbens, and cingulum bundle (Fig. 1E,G). Sub-therapeutic stimulation through contact \#3 (4 mA) activated the same general pathways, but only 2,465 axon models. Non-therapeutic stimulation through contact \#1 (8 mA) activated 2,196 axon models which were associated with the more posterior/caudal aspects of the vmPFC (i.e. BA32) and accumbens. However, contact \#1 stimulation did not activate the cingulum bundle or the rostral vmPFC (Fig. 1F,H).

\section{Discussion}

We present a patient-specific DBS TAM where both the DWI-based tractography and electric field models were derived from imaging data acquired directly from the patient. Previous neuropsychiatric DBS tractography studies relied on DWI atlas brains that may not account for the nuances of the patient's specific neuroanatomy $[3,4,6]$. In addition, probabilistic tractography allowed us for the first time to couple our DBS axonal activation predictions with inferences of cortical and sub-cortical connectivity.

The SCCWM DBS target carries multiple fibers connecting the frontal cortex, cingulate, accumbens, thalamus, amygdala, hippocampus, and brainstem [5]. Functional imaging results confirm widespread network changes associated with DBS induced antidepressant effects [1]. Therefore, it is unlikely that a single axonal pathway fully accounts for the therapeutic effects of SCCWM DBS. Our case report suggests that activation of a critical mass of a unique combination of cortical, sub-cortical, and cingulate pathways may be necessary for therapeutic benefit.

We propose that patient-specific DBS TAMs represent a useful technique to compare pathway activation in response to DBS at different parameter settings. In addition, DBS TAMs created for populations of patients will enable comparison of pathway activation in responders vs. non-responders. Such efforts could assist in the definition of target pathways for therapeutic benefit in the range of DBS clinical applications. However, validation of 
TAM predictions will require integration of multiple experimental modalities such as functional imaging, electrophysiology, and anatomical tract tracing.

\section{Acknowledgments}

Dana Foundation (HSM), Woodruff Fund (HSM), Stanley Foundation (HSM), National Institutes of Health R01 NS059736 (CCM).

The authors thank Kabilar Gunalan for assistance with the computer simulations.

\section{References}

1. Mayberg HS, Lozano AM, Voon V, McNeely HE, Seminowicz D, Hamani C, Schwalb JM, Kennedy SH. Deep brain stimulation for treatment-resistant depression. Neuron. 2005; 45(5):65160. [PubMed: 15748841]

2. Holtzheimer PE, Kelley ME, Gross RE, Filkowski MM, Garlow SJ, Barrocas A, Wint D, Craighead MC, Kozarsky J, Chismar R, Moreines JL, Mewes K, Riva Posse P, Gutman DA, Mayberg HS. Subcallosal Cingulate Deep Brain Stimulation for Treatment-Resistant Unipolar and Bipolar Depression. Arch Gen Psychiatry. 2012; 69(2):150-8. [PubMed: 22213770]

3. Johansen-Berg H, Gutman DA, Behrens TE, Matthews PM, Rushworth MF, Katz E, Lozano AM, Mayberg HS. Anatomical connectivity of the subgenual cingulate region targeted with deep brain stimulation for treatment-resistant depression. Cereb Cortex. 2008; 18(6):1374-83. [PubMed: 17928332]

4. Gutman DA, Holtzheimer PE, Behrens TE, Johansen-Berg H, Mayberg HS. A tractography analysis of two deep brain stimulation white matter targets for depression. Biol Psychiatry. 2009; 65(4):27682. [PubMed: 19013554]

5. Lehman JF, Greenberg BD, McIntyre CC, Rasmussen SA, Haber SN. Rules ventral prefrontal cortical axons use to reach their targets: implications for diffusion tensor imaging tractography and deep brain stimulation for psychiatric illness. J Neurosci. 2011; 31(28):10392-402. [PubMed: 21753016]

6. Lujan JL, Chaturvedi A, Malone DA, Rezai AR, Machado AG, McIntyre CC. Axonal pathways linked to therapeutic and nontherapeutic outcomes during psychiatric deep brain stimulation. Hum Brain Mapp. 2012; 33(4):958-68. [PubMed: 21520343]

7. Jenkinson M, Beckmann CF, Behrens TE, Woolrich MW, Smith SM. FSL. NeuroImage. 2012; 62(2):782-90. [PubMed: 21979382]

8. Chaturvedi A, Butson CR, Lempka SF, Cooper SE, McIntyre CC. Patient-specific models of deep brain stimulation: influence of field model complexity on neural activation predictions. Brain Stimul. 2010; 3(2):65-7. [PubMed: 20607090]

9. McIntyre CC, Richardson AG, Grill WM. Modeling the excitability of mammalian nerve fibers: influence of afterpotentials on the recovery cycle. J Neurophysiol. 2002; 87(2):995-1006. [PubMed: 11826063]

10. Butson CR, Cooper SE, Henderson JM, McIntyre CC. Patient-specific analysis of the volume of tissue activated during deep brain stimulation. NeuroImage. 2007; 15;34(2):661-70.

11. Tuch DS, Wedeen VJ, Dale AM, George JS, Belliveau JW. Conductivity tensor mapping of the human brain using diffusion tensor MRI. Proc Natl Acad Sci U S A. 2001; 98(20):11697-701. [PubMed: 11573005]

12. McNeal DR. Analysis of a model for excitation of myelinated nerve. IEEE Trans Biomed Eng. 1976; 23(4):329-37. [PubMed: 1278925] 

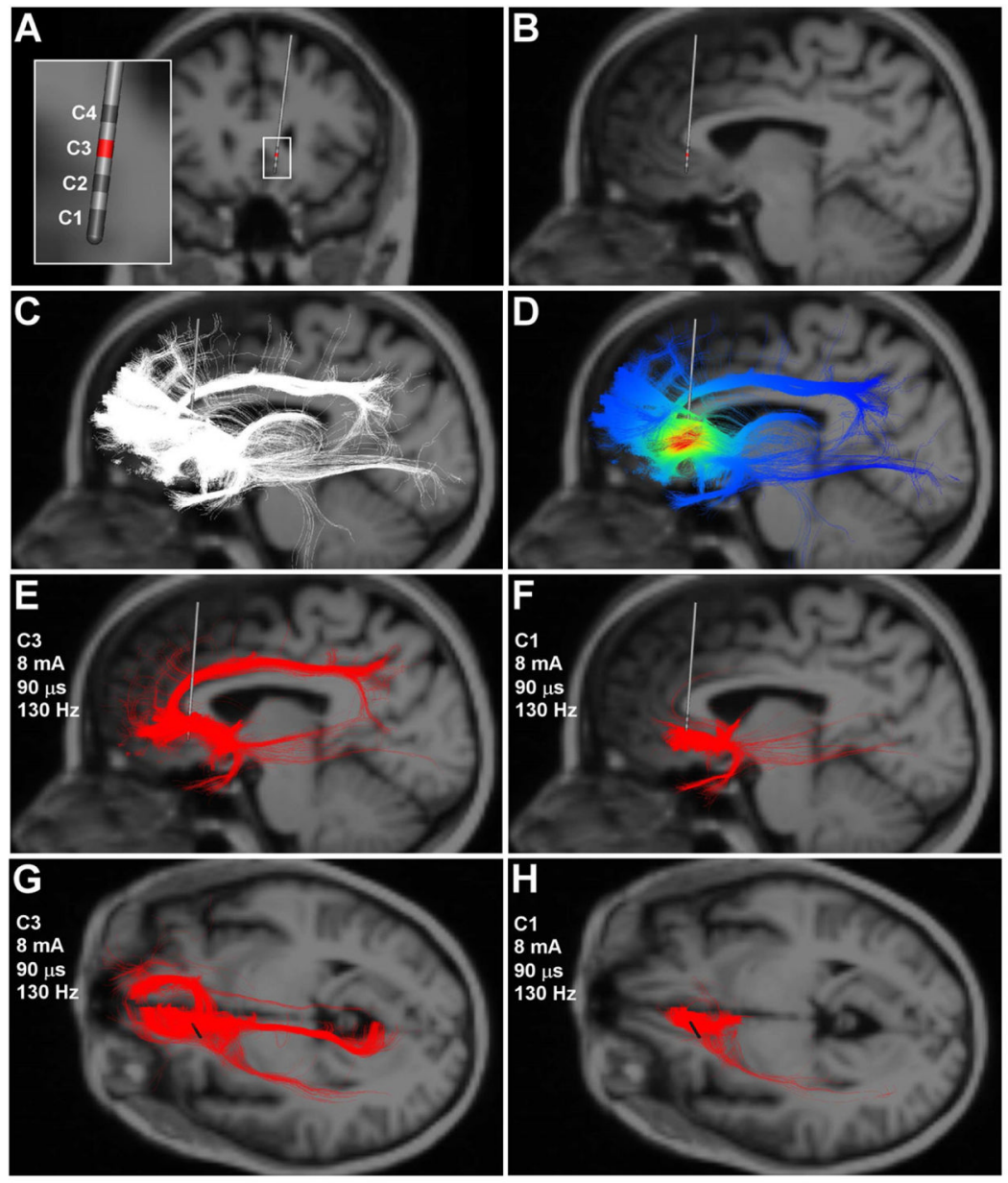

\section{Figure 1.}

Tractography-activation models. A) Coronal image of the DBS electrode location. Inset shows the 4 contact lead and the respective contact numbers. Stimulation through contact 3 resulted in the best clinical outcomes. B) Sagittal view of the DBS electrode location. Note that the MRI background image is $3 \mathrm{~mm}$ behind the tip of the DBS electrode as not to obscure the tractography results which project in $3 \mathrm{D}$ around the electrode in following panels. C) White streamlines represent axon model trajectories passing by the DBS electrode. D) Extracellular voltage distribution imposed upon each axon model from stimulation at contact 3. E,G) Axon models (red streamlines) directly activated by therapeutic stimulation. F,H) Axon models directly activated by non-therapeutic stimulation. 